\title{
Three-dimensional echocardiography-guided beating-heart surgery without cardiopulmonary bypass: A feasibility study
}

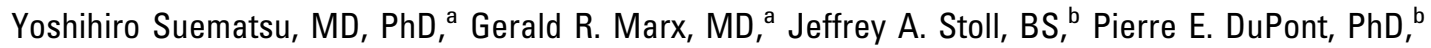
Robin 0. Cleveland, PhD, ${ }^{\mathrm{b}}$ Robert D. Howe, PhD, ${ }^{\mathrm{c}}$ John K. Triedman, MD, ${ }^{\mathrm{a}}$ Tomislav Mihaljevic, MD, ${ }^{\mathrm{a}}$ Bassem N. Mora, MD, ${ }^{a}$ Bernie J. Savord, MS, ${ }^{d}$ Ivan S. Salgo, MD, MS, ${ }^{d}$ and Pedro J. del Nido, MD

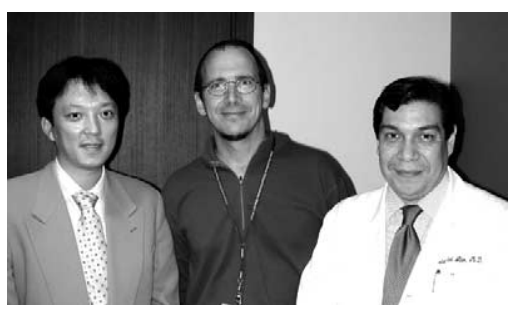

Suematsu, Triedman, del Nido

\begin{abstract}
From the Departments of Cardiac Surgery and Cardiology, ${ }^{\mathrm{a}}$ Children's Hospital-Boston and Brigham and Women's Hospital, Harvard Medical School, Boston, Mass; the Department of Aerospace and Mechanical Engineering, , Boston University, Boston, Mass; the (3) Division of Engineering and Applied Sciences, ${ }^{\mathrm{c}}$ Harvard University, Cambridge, Mass; and Philips Medical Systems, ${ }^{\mathrm{d}}$ Andover, Mass.
\end{abstract}

BJS and ISS are employees of Philips Medical Systems, who provide technical guidance.

Supported in part by National Institutes of Health grants HL71128 and HL 73647 (PJD) and by the Japan Society for the Promotion of Science (YS). The authors acknowledge the instrumental support of Yoshimasa Tochimura, Takashi Ina (Research and Development Laboratory, Mani, Inc, Tochigi, Japan), and Katsuya Miyagawa (Research and Development Laboratory, Nipro Co, Shiga, Japan)

Read at the Eighty-fourth Annual Meeting of The American Association for Thoracic Surgery, Toronto, Ontario, Canada, April 25-28, 2004.

Received for publication April 13, 2004; revisions received May 21, 2004; accepted for publication June 1, 2004.

Address for reprints: Pedro J. del Nido, MD, Department of Cardiac Surgery, Children's Hospital-Boston, 300 Longwood Ave, Boston, MA 02115 (E-mail: pedro.delnido@ tch.harvard.edu).

J Thorac Cardiovasc Surg 2004;128:579-87

0022-5223/\$30.00

Copyright (C) 2004 by The American Association for Thoracic Surgery

doi:10.1016/j.jtcvs.2004.06.011
Background: There is no current acceptable approach for intracardiac beating-heart interventions. We have adapted real-time 3-dimensional echocardiography with specialized instrumentation to facilitate beating-heart repair of atrial septal defects and mitral valve plasty to investigate the feasibility of real-time 3-dimensional echocardiography-guided cardiac surgery.

Methods: In experiment I a modified real-time 3-dimensional echocardiography system with $\mathrm{x} 4$ matrix transducer was compared with 2-dimensional echocardiography in the performance of common surgical tasks. Completion times, deviation from an ideal trajectory, and an echogenic target were measured. In experiment II porcine atrial septal defects were closed with an original semiautomatic suturing device $(n=4)$ and with a 5 -mm endoscopic stapler and a pericardial or polytetrafluoroethylene patch $(n=4)$. In experiment III a pulsatile porcine mitral valve model was developed, and suture placement through the anterior and posterior mitral leaflets was performed $(n=8)$. During all experiments, the operator was blinded to the target and operated on only with ultrasonic guidance.

Results: In experiment I, compared with 2-dimensional echocardiographic guidance, completion times improved by $21 \%(P<.01)$ with high-trajectory accuracy, and suture deviation was significantly smaller (2-dimensional echocardiography, $5.4 \pm$ $2.7 \mathrm{~mm}$; 3-dimensional echocardiography, $1.7 \pm 0.7 \mathrm{~mm} ; P<.05)$ in real-time 3-dimensional echocardiography-guided tasks. In experiments II and III in both atrial septal defect closure and mitral valve plasty, real-time 3-dimensional echocardiography provided satisfactory images and sufficient anatomic detail for suturing and patch deployment. All surgical tasks were successfully performed with accuracy.

Conclusions: Real-time 3-dimensional echocardiography provides adequate imaging and anatomic detail to act as a sole guide for surgical task performance. These initial experiments demonstrate the feasibility of beating-heart direct or patch closure of atrial septal defects and mitral valve plasty without cardiopulmonary bypass.

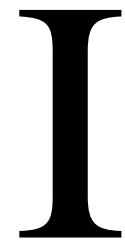
ntracardiac surgery has challenged surgeons and researchers since the pioneers of modern cardiac surgery first performed transatrial valvuloplasty ${ }^{1,2}$ and closure of atrial septal defects (ASDs) in a beating heart. ${ }^{3}$ However, these first beating-heart procedures were hampered by inadequate visualization of intracardiac structures, which led to suboptimal results. These approaches fell into disfavor when cardiopulmonary bypass (CPB), which allowed direct visualization of intracardiac structures, was introduced. Nevertheless, CPB is widely recognized as having a number of adverse effects, including generation of microemboli and an inflammatory response associated with increased cytokine production and complement activation, which together can result 
in neurologic dysfunction in adults and neurodevelopmental dysfunction in children., ${ }^{4,5}$

Furthermore, the repairs during reconstructive surgical procedures inside the heart performed with CPB and cardiac arrest are carried out inside a relaxed heart, which can be easily distorted by surgical retractors. This makes evaluation of the geometry relative to the reconstructive ideal quite challenging, particularly for mitral valve surgery. ${ }^{6}$ Also, because of the nonphysiologic state of the empty nonbeating heart during repair, full assessment of the quality of the surgical repair cannot be completed until the heart is closed and CPB is discontinued.

Real-time three-dimensional echocardiography (RT3DE) has recently been developed and provides clinicians and surgeons with a new perspective for visualizing the heart noninvasively without electrocardiographic or respiratory gating. First, von Ramm and colleagues $^{7}$ reported 2-dimensional (2D) crystal arrays for RT3DE in 1990. A new probe has now been developed that creates a fully sampled 2D array and allows 3-dimensional (3D) imaging with instantaneous, online, volume-rendered reconstruction, direct manipulation of thresholding, and planes cut on the ultrasound unit. This new 3D probe has great potential for expanded application not only to medical diagnosis but also for imageguided surgical intervention. The primary purpose of this study was to determine whether RT3DE with the 2D matrix array probe provides better image guidance than conventional 2D echocardiography (2DE) for surgical interventions. The second purpose was to experimentally investigate the feasibility of beating intracardiac surgery, including off-pump ASD closure and off-pump mitral valve plasty (MVP), monitored by RT3DE in vitro in a water tank experiment and to evaluate new surgical devices.

\section{Materials and Methods}

\section{Echocardiographic Equipment}

RT3DE was performed by using the $\mathrm{x} 4$ matrix transducer on a Sonos 7500 system (Philips Medical Systems, Andover, Mass). The transducer operates in a broadband 2- to $4-\mathrm{MHz}$ range and scans a 3D volume by electronically steering the acoustic beam with a matrix of approximately 3000 transducer elements and associated electronics that allow scanning of a $64^{\circ} \times 64^{\circ}$ pyramidal volume in real time at up to 28 frames per second. A schematic diagram of the principle of volume mode imaging is shown in Figure 1. The Sonos 7500 base system volume renders the data in any viewing orientation desired, also at a $28-\mathrm{Hz}$ frame rate, and the orientation of the target object on the screen can be controlled with a roller ball. The image processing and rendering platform is based on a dual 2.2-GHz Pentium 4 processor PC, which supports multiple imaging modalities, including conventional B-mode 2DE, 2D color-flow Doppler imaging, biplanar 2DE, and several realtime volume-rendering modes.

\section{Custom Tank}

A custom tank was prepared for this study to evaluate surgical performance under simulated clinical image-guidance conditions. The tank consists of an acrylic reservoir covered by an opaque dome through which surgical instruments are inserted. The bottom of the tank is lined with an acoustic polymer (Shin-Etsu Polymer Co, Ltd, Tokyo, Japan) to prevent echocardiographic beam reflection from the tank. Degassed double-deionized $\mathrm{H}_{2} \mathrm{O}$ serves as the imaging medium inside the testing tank.

\section{Surgical Devices}

A semiautomatic suturing device we developed for off-pump intracardiac surgery was used as previously described. ${ }^{8,9}$ A thread (4-0 silk) is attached to one end of the needle, which has a conical point at the other end. The bottom of the needle is affixed in a niche on the tip of the proximal jaw of the semiautomatic suture device by pulling the thread down with one hand. The jaws close after the needle is passed through the tissue to be fixed. When the jaw is slowly opened again, the tip of the needle is automatically grasped by the distal jaw, which has a resilient slit. When the device is gently moved off the tissue, the thread should have penetrated the tissue appropriately. After the device is pulled outside the cardiac cavity with the needle, the same action is repeated to the opposing edge of tissue. The device is $5 \mathrm{~mm}$ in diameter. In addition to the semiautomatic suturing device, we also used a commercially available stapler (Tacker; Autosuture, Norwalk, Conn), which has a $5 \mathrm{~mm}$-diameter shaft, delivers spiralshaped titanium tacks, and has a multiuse handle, for the purpose of surgical approximation.

We used a commercially available knot pusher (Olympus Co, Tokyo, Japan) for ligation of the thread in minimally invasive cardiac surgery. A suture-cutting device consisting of a shaft, cutting edge, and handle was used to cut the suture. ${ }^{9}$ The shaft consists of inner and outer tubes, and the outer diameter of the outer tube is $2.5 \mathrm{~mm}$. The inner shaft has the cutting edge, which is used to insert and cut the thread. The handle is connected to the end of the shaft and is used to turn the inner tube. The thread is cut by placing it between the inner and outer cutting edges.

Finally, to deploy a patch for ASD closure, an original patchopener device, which consists of a self-expanding ring of nitinol and a grip, was used (Figure 2). Autologous pericardium or a 0.1-mm polytetrafluoroethylene patch (Gore-Tex, W. L. Gore \& Associates, Inc, Flagstaff, Ariz) was appropriately trimmed and then sutured along the ring with 8-0 Prolene sutures (Ethicon Inc, Somerville, NJ). This device can be delivered through a long sheath ( $5 \mathrm{~mm}$ in diameter), and a nitinol ring with autologous pericardium is advanced out the end of the sheath and allowed to expand.

\section{DE Versus RT3DE Images for Guiding Surgical}

\section{Tasks}

Because 2DE provides only limited spatial orientation data, its use for guiding interventional procedures has been limited to procedures in which a rigid tool can be maintained in the plane of the ultrasound image at all times. ${ }^{10}$ The following study was performed to determine the potential applicability of RT3DE to guiding basic surgical tasks. 


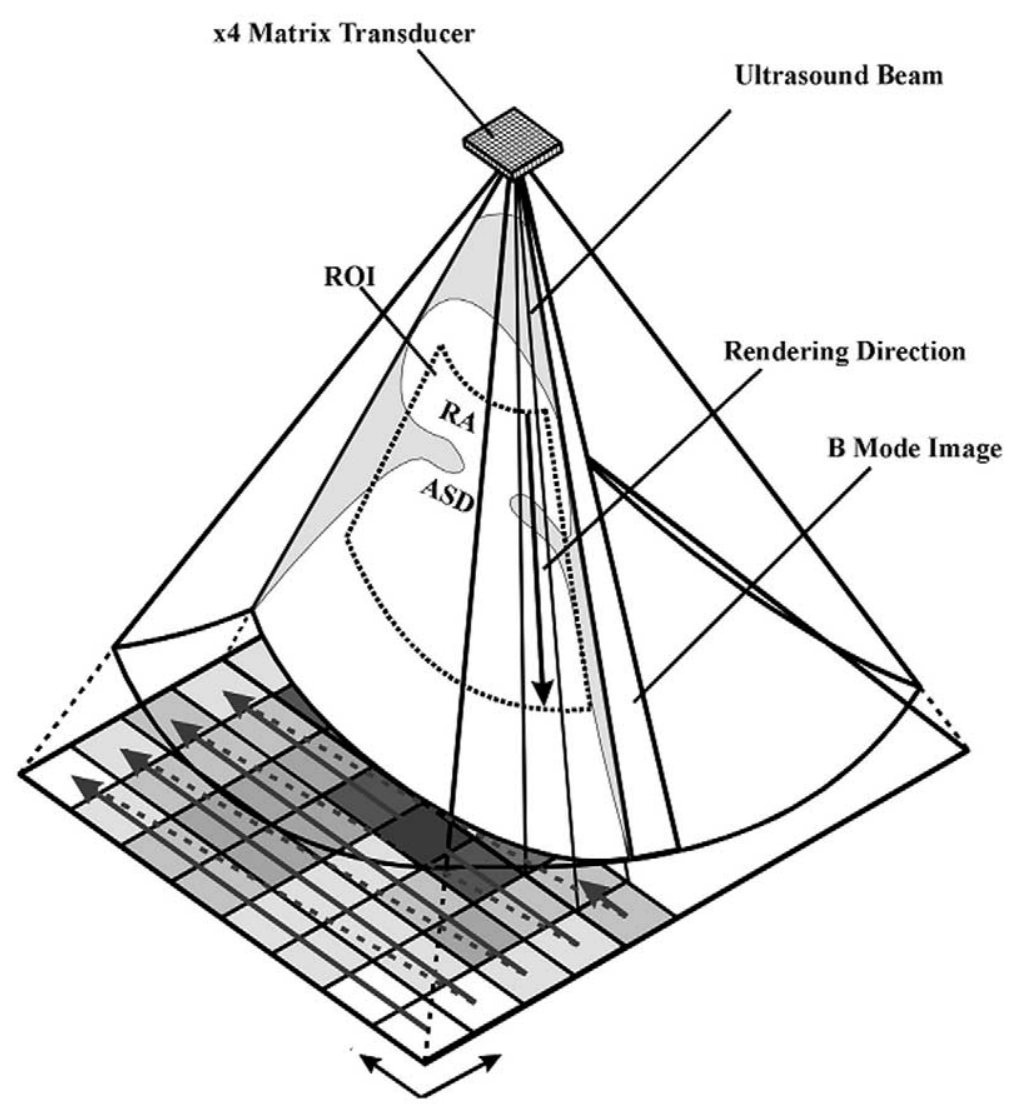

Figure 1. Schematic diagram illustrating the principle of volume mode imaging. Matrix phased array uses a matrix of crystals that can collect a pyramidal volume of data 20 times per second in real time. Appropriate 3-dimensional echocardiographic images of the ASD are displayed by means of user manipulation of a region of interest (ROI) that includes the right atrium $(R A)$ and the left atrium.

Stapling with Tacker was performed in the water-filled tank by using either 2DE or RT3DE performed by an experienced ultrasonographer for guidance $(\mathrm{n}=10)$. The operator was blinded to the target and operated on only under ultrasonic guidance. Task completion time and deviation of the suture from an echogenic target (in millimeters) were measured. Tool deviation from an ideal trajectory as an indicator of performance accuracy (in centimeters) was also measured with an electromagnetic tracker mounted on the surgical tool (Flock of Birds; Ascension Technologies, Burlington, Vt), as previously described. ${ }^{11}$ The ideal trajectory is a straight line from the starting point to the target, with total trajectory deviation distance given by equation 1 .

$$
\mathrm{D}_{\text {mean }}=\frac{1}{n}\left(\sum_{j} h i+\sum_{j} h j \sin \theta_{j}+\sum_{k} h_{k}\right)
$$

Dmean is the mean deviation distance measured as the distance between each data point acquired and either the target (hi or hk) or the liner trajectory between the starting point and the target (hj $\sin \theta \mathrm{j}$ ). Mean deviation is obtained by dividing $\mathrm{D}$ by the number of data points taken to obtain a measurement that is independent of task performance time.

\section{Ex Vivo ASD Closure and Mitral Valve Repair}

The operator was again blinded to the target during the experiment and operated on only under ultrasonic guidance, the same as in the above tank study.

\section{ASD Closure}

The intra-atrial septa from 8 slaughterhouse pig hearts were mounted in the water tank, and the septum secundum regions were excised to create an ASD $(8.5 \pm 2.5 \mathrm{~mm}$; approximately 5-12 $\mathrm{mm}$ ). Four defects were closed with interrupted sutures by means of a semiautomatic suturing device, and the other 4 were closed with a patch by using the Tacker and patch-opener device (patch materials: autologous pericardium $[\mathrm{n}=2]$ and $0.1-\mathrm{mm}$ polytetrafluoroethylene sheet $[n=2])$. The thread of the interrupted sutures was cut with the suture-cutting system. After completion of the procedure, suture placement was assessed, and accuracy was quantified.

\section{Mitral Valve Repair}

An in vitro pulsatile model of the mitral valve was used to assess the feasibility of image-guided mitral valve repair. The valved 

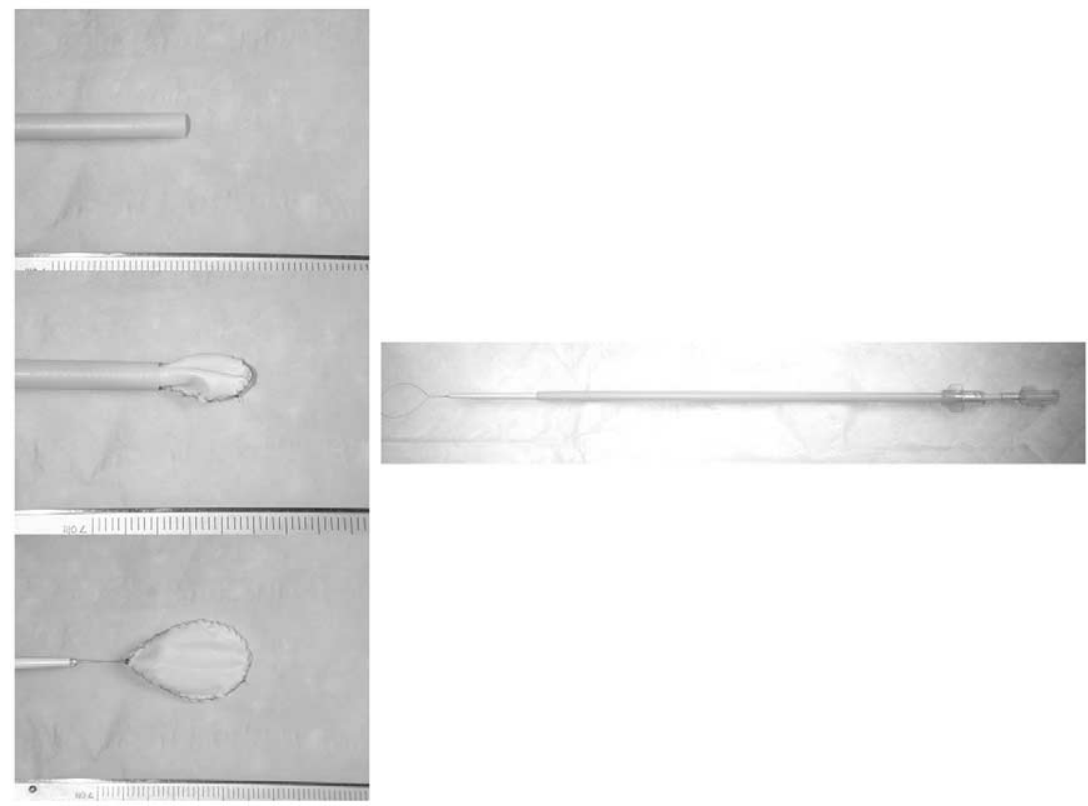

Figure 2. The patch-deployment device, which consists of a self-expanding nitinol ring and a grip, was originally developed to navigate patches to the ASDs. Left, A $0.1-\mathrm{mm}$ polytetrafluoroethylene patch has been sutured to the nitinol ring.

TABLE 1. Results

\begin{tabular}{|c|c|c|c|c|c|c|c|c|c|}
\hline No. & Model & $\begin{array}{c}\text { Size of } \\
\text { ASD } \\
(\mathrm{mm})\end{array}$ & Surgical method & $\begin{array}{l}\text { No. of } \\
\text { sutures }\end{array}$ & $\begin{array}{c}\text { Suture } \\
\text { deployment }\end{array}$ & $\begin{array}{l}\text { Patch } \\
\text { fixation }\end{array}$ & $\begin{array}{l}\text { Tissue } \\
\text { trauma }\end{array}$ & $\begin{array}{c}\text { Knot } \\
\text { tension }\end{array}$ & $\begin{array}{c}\text { Suture } \\
\text { placement } \\
>2 \mathrm{~mm} \\
\text { from edges }\end{array}$ \\
\hline 1 & ASD & 5 & $\mathrm{DC}$ & 1 & + & NA & None & + & + \\
\hline 2 & ASD & 8 & $\mathrm{DC}$ & 2 & + & NA & None & + & + \\
\hline 3 & ASD & 5 & $\mathrm{DC}$ & 1 & + & NA & None & + & + \\
\hline 4 & ASD & 10 & $\mathrm{DC}$ & 3 & + & NA & None & + & + \\
\hline 5 & ASD & 10 & $P C(A P, 15 \mathrm{~mm})$ & 4 & + & + & None & NA & + \\
\hline 6 & ASD & 8 & $P C(A P, 15 \mathrm{~mm})$ & 5 & 1: misplace & + & None & NA & + \\
\hline 7 & ASD & 12 & PC (PTFE, 15 mm) & 4 & + & + & None & NA & + \\
\hline 8 & ASD & 10 & PC (PTFE, $15 \mathrm{~mm}$ ) & 4 & + & + & None & NA & + \\
\hline 9 & $\mathrm{MR}$ & NA & DOR & 1 & + & NA & None & + & + \\
\hline 10 & MR & NA & DOR & 1 & + & NA & None & + & + \\
\hline 11 & MR & NA & DOR & 2 & + & NA & None & + & + \\
\hline 12 & MR & NA & DOR & 2 & + & NA & None & + & + \\
\hline 13 & MR & NA & PCR & 1 & + & NA & None & + & + \\
\hline 14 & MR & NA & PCR & 1 & + & NA & None & + & + \\
\hline 15 & MR & NA & PCR & 1 & + & NA & None & + & + \\
\hline 16 & MR & NA & PCR & 1 & + & NA & None & + & + \\
\hline
\end{tabular}

$\overline{A S D}$, Atrial septal defect; $D C$, direct closure; +, acceptable; NA, not applicable; $P C$, patch closure; $A P$, autologous pericardial patch; $P T F E$, polytetrafluoroethylene patch; $M R$, mitral repair; $D O R$, double-orifice repair, $P C R$, paracommissural repair.

chamber was constructed with mitral valves from 8 slaughterhouse pig hearts and commercially available $2-\mathrm{mm}$ silicone rubber sheeting to prevent image deterioration caused by side-lobe artifacts from the valve cage itself. The valves were suspended in the chamber, with the annulus of the valve sewn to one ring and the papillary muscles anchored. The cage was also connected to an outflow valve and a $45-\mathrm{mL}$ rubber pump. The pump was manually controlled, and a wide range of heart rates can be simulated. Mitral 
(1)

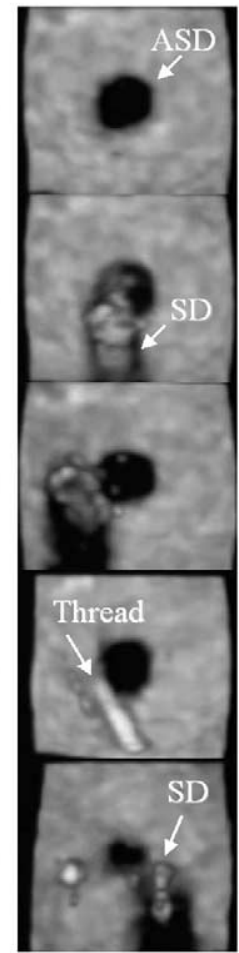

(6)

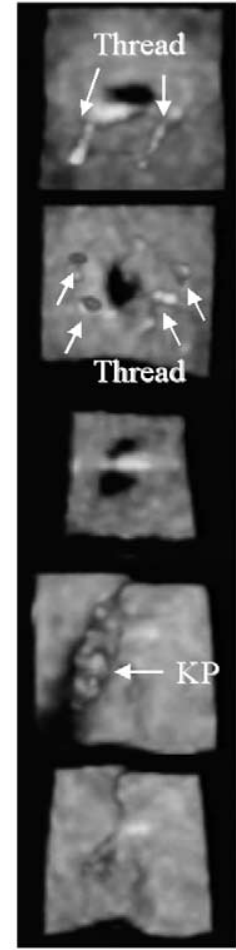

Figure 3. Sequential RT3DE images showing direct closure of the ASD with the semiautomatic suturing device (SD). A thread that has been passed through the rim of the ASD can be seen (4-7), and the ASD was completely closed with a knot pusher (KP).

valve regurgitation was created at the center $(n=4)$ or the paracommissure site $(n=4)$ of all valves by loosening or releasing the anterior or posterior papillary muscle to mimic prolapse of the mitral leaflet. Heart rate was kept constant at 60 cycles per minute throughout the experiment. The edge-to-edge method was chosen as the surgical maneuver for MVP because of its simplicity and the fact that it requires accurate suture placement in moving leaflets for task completion. ${ }^{12,13}$ Double-orifice repair and paracommissural repair by means of the edge-to-edge method were simulated by approximating the free edges of the leaflets at the site of regurgitation with interrupted sutures by using the semiautomatic suturing device, each suture was tied with a knot pusher, and the thread was cut with the suture-cutting system, as described above. Valve function was assessed by means of 3D and 2D imaging of leaflet coaptation. Proper placement of the sutures was confirmed at the end of the experiment.

\section{Statistical Analysis}

Statistical analysis was performed with the Stat View (Version 5) software package (SAS Institute, Inc, Cary, NC). Data are expressed as means \pm SDs. Surface processing was analyzed by means of 1-way analysis of variance, followed by the Student $t$ test for unpaired data with the Bonferroni correction, and the comparison between 2DE and RT3DE images for guiding surgical tasks was analyzed by using the Student $t$ test.

(1)

(2)

(3)

(4)

(5)

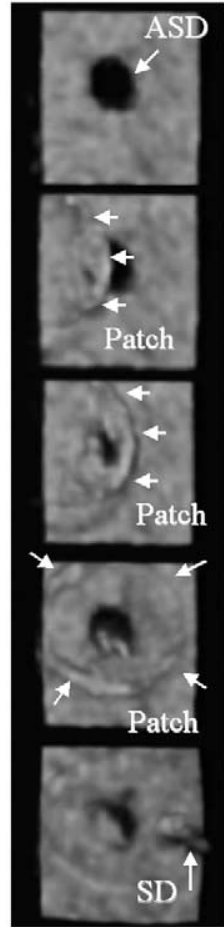

(6)

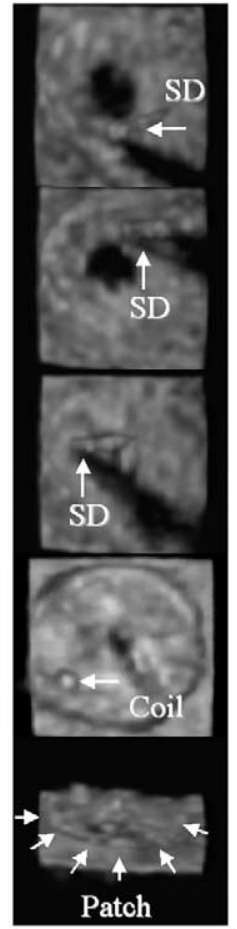

Figure 4. Sequential RT3DE images showing patch closure of the ASD with the patch-opener device and Tacker (SD). Arrows indicate the rim of the patch $(2-4,10)$. The location of the ASD hole can be seen through the patch.

\section{Results}

2DE Versus RT3DE Images for Guiding Surgical

Tasks

Compared with 2DE guidance, suturing completion times improved 21\% (47.4 \pm 10.9 seconds and $9.9 \pm 3.1$ seconds, $P<.01)$ with RT3DE because the spatial orientation of the ASD and suturing devices were more easily observed with RT3DE. Tool-tip trajectory tracking for suturing navigation demonstrated greater navigational accuracy measured by means of trajectory deviation $(10.1 \pm 2.3 \mathrm{~cm}$ and $5.1 \pm 1.8$ $\mathrm{cm}, P<.05$ ), and the deviation of the suture from the echogenic target was significantly smaller with RT3DE guidance than with $2 \mathrm{DE}$ guidance $(1.7 \pm 0.7 \mathrm{~mm}$ and $5.4 \pm$ $2.7 \mathrm{~mm}, P<.05$ ).

\section{Ex Vivo ASD Closure and Mitral Valve Repair}

ASD closure. The results for each procedure, including ASD direct and patch closure, are summarized in Table 1, and representative echocardiographic images of ASD direct closure and patch closure are shown in Figures 3 and 4. The best images were obtained with the ultrasonic transducer at distances of approximately 4 to $6 \mathrm{~cm}$ from the target. The echocardiographic shadows produced on the surface of the atrial septal tissue by the instruments were found to be useful for recognizing the distance from the target, even on 
(1)

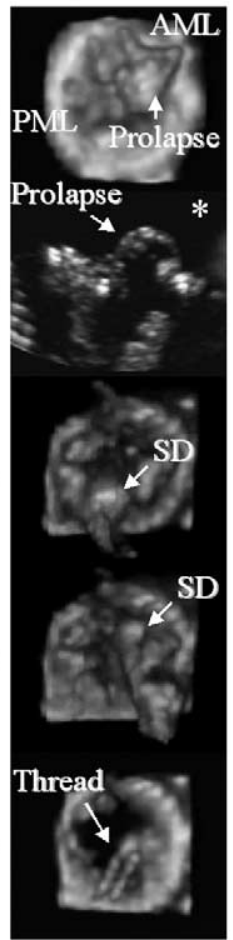

(6)

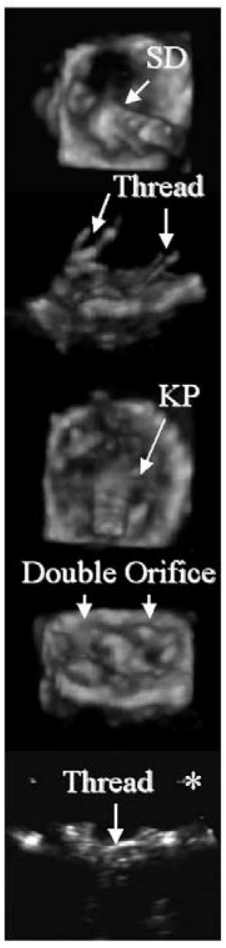

(1)

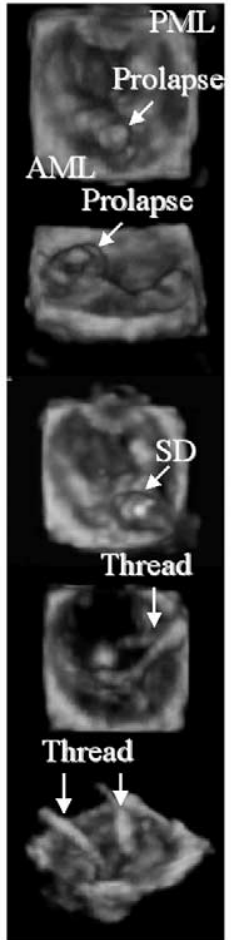

(6)

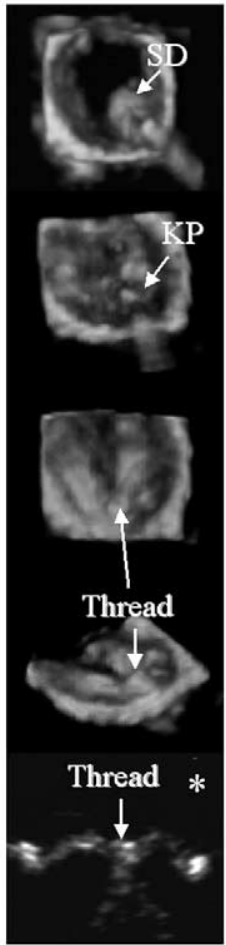

Figure 5. RT3DE images showing double-orifice repair of the mitral valve by using the edge-to-edge method with the semiautomatic suturing device (SD). One end of a thread has been passed through the prolapsed part of anterior mitral leaflet (AML; $3-5)$, and the other end has been passed through the corresponding part of the posterior mitral leaflet (PML; 6, 7). The thread was completely tied with a knot pusher (KP; 8$)$, and a double orifice was created (9). *Two-dimensional echocardiographic image.

the 2D-rendered image of the echocardiographic system. Both the semiautomatic suturing device and the Tacker functioned satisfactorily in all 8 ASD procedures, and the spatial orientation of the ASD, the atrial septal tissue, and both suturing devices was easily recognized with RT3DE. The semiautomatic suturing device successfully penetrated the ASD rim tissue with needle and suture in every ASD direct closure, and successful suture deployment was confirmed after the procedure. The number of sutures placed for closure was $1.8 \pm 1.0$ (range, 1-3). The needles consistently penetrated the ASD tissue approximately 3 to $4 \mathrm{~mm}$ from the free edge of the tissue margin, and the knots were also consistently tied and cut. All ASDs were successfully closed. In the patch-closure experiments the patches were easily deployed in a suitable position in all cases with RT3DE. By means of appropriate adjustment of the gain setting of the echocardiographic probe, the patch rim and ASD hole were visualized simultaneously. The Tacker successfully penetrated both the ASD rim tissue and patch material, and successful deployment of coil was confirmed
Figure 6. RT3DE images showing paracommissural repair of the mitral valve by using the edge-to-edge method with the semiautomatic suturing device (SD). One end of a thread has been passed through the prolapsed part of anterior mitral leaflet (AML; 3-5). After passing the other end of the thread through the corresponding part of the posterior mitral leaflet (PML; 6), the thread was tied by using a knot pusher (KP; 7-10). *Two-dimensional echocardiographic image.

after the procedure, except for one coil, which was misplaced and only penetrated the atrial tissue. The number of sutures was $4.3 \pm 0.5$ (range, $4-5$ ). When placed over the patch, the coils consistently penetrated the ASD tissue and patch materials, and all ASDs were successfully closed. No collateral tissue injuries were observed with either method.

Mitral valve repair. The results of each procedure are summarized in Table 1, and representative echocardiographic images of both double-orifice repair and paracommissural repair are shown in Figures 5 and 6. As in ASD direct closure, the semiautomatic suturing device functioned satisfactorily in all 8 mitral valve procedures, and the spatial orientation of the mitral leaflets, the commissures, and the device was easily recognized by means of RT3DE. The device successfully captured each leaflet, and successful suture deployment was confirmed after the procedure. The number of sutures was $1.8 \pm 0.5$ (range, 1-2). The needles consistently penetrated the mitral leaflet approximately 3 to $4 \mathrm{~mm}$ from the free edge of the tissue margin, the same as with direct ASD closure, and the knots were also consis- 
tently tied and cut. Both double-orifice repair and paracommissural repair could be successfully performed (each $\mathrm{n}=$ 4). No collateral tissue injuries, such as leaflet trauma, were observed.

\section{Discussion}

The introduction of CPB and subsequent technologic advances have made cardiac surgery safe and reliable, and almost all surgeons have used CPB exclusively for these repairs since the 1960s. Nevertheless, CPB is widely recognized as giving rise to a number of adverse effects, such as blood coagulation abnormalities, changes in erythrocyte and plasma proteins, and a nonspecific inflammatory response. Gas and particulate emboli and various vasoactive and otherwise biologically active substances are also produced by contact between blood and foreign surfaces during $\mathrm{CPB}$ and can sometimes result in pulmonary, myocardial, and neurologic dysfunction. ${ }^{4,5}$ Coronary artery bypass grafting without CPB (off-pump coronary artery bypass grafting) has recently become a more widely accepted alternative technique for myocardial revascularization in patients with poor ventricular function who might not tolerate $\mathrm{CPB}$ and in patients with complications in whom CPB, hypothermia, and aortic cannulation are undesirable. ${ }^{14,15}$ However, because of the presence of blood and heart motion, imaging the inside of the heart is more difficult than imaging the inside of the epicardial coronary arteries.

Interventions guided by echocardiography and fluoroscopy, such as catheter-based ASD closure and balloon mitral commissurotomy, are commonly performed in the cardiac catheterization laboratory, ${ }^{16-18}$ and the concept of image-guided intervention in cardiac surgery is not new. The use of a cardioscope to guide intracardiac surgery had already been described in the 1920s, although the visualization of intracardiac structures was suboptimal. ${ }^{19,20}$ Sogawa and associates ${ }^{21}$ and Downing and coworkers ${ }^{22}$ recently developed direct image-guided approaches, including optical imaging with an endocardioscope for experimental ASD closure and transesophageal echocardiography-guided suturing in experimental mitral valve surgery. However, they described inadequate visualization of the surgical target as a significant limitation of such approaches.

A variety of 3D echocardiography systems have been developed during the past decade, and they provide clinicians and surgeons with new perspectives for visualizing the heart. ${ }^{23,24} \mathrm{RT} 3 \mathrm{DE}$, in particular, provides real-time visualization of the heart noninvasively without electrocardiographic or respiratory gating because all planes are imaged simultaneously. ${ }^{25}$ Accordingly, we used this new system as the sole guiding method to generate images with sufficient spatial and temporal resolution for beating-heart surgery.

The RT3DE system we used consists of a novel transducer and a custom image processing and rendering plat- form. The imaging probe is a handheld transducer containing approximately 3000 active piezoelectric crystals, and our colleagues (BJS and ISS) developed a method of creating a fully-sampled 2D array. This system also enables scanning of a $64^{\circ} \times 64^{\circ}$ pyramidal volume in real time at up to 28 frames per second. In addition, the image of the target object on the screen can be easily manipulated, and the operator can view the target from any angle without moving the imaging transducer. Because of this, accurate positioning of the surgical instruments and patch material was confirmed by using multiple views in our tank study.

Although it might be possible to perform surgical intervention with $2 \mathrm{D}$ images alone, spatial relationships cannot be grasped instantaneously, and obtaining favorable images in which both the target tissue and the surgical device are monitored simultaneously would be time-consuming, making it impractical as a real-time guide. Downing and coworkers $^{22}$ reported a successful suture rate of $75 \%$ and a mean distance from the ideal position at the midportion of the leaflet of $8.5 \pm 5.0 \mathrm{~mm}$ in their experimental beatingheart mitral valve operations monitored by means of $2 \mathrm{DE}$ alone. This was not considered accurate enough and would not be acceptable in clinical situations because the target structures were small. The results of our study show that compared with 2DE guidance, completion times significantly improved by $21 \%(P<.01)$ with high-trajectory accuracy, and suture deviation was significantly smaller (2DE, $5.4 \pm 2.7 \mathrm{~mm}$; 3DE, $1.7 \pm 0.7 \mathrm{~mm} ; P<.05$ ) in the RT3DE-guided tasks. We therefore consider the RT3DE to allow the surgeon's view to be obtained in real time and to allow the surgeon to perform surgical interventions in the same manner as endoscopic surgery.

Although current 3D computed tomography and magnetic resonance images might provide high-resolution images of the heart, both involve prolonged imaging time, high equipment costs, and special facilities. The immobility of these systems also limits their clinical use in the operating room. Furthermore, the metallic composition of the advanced medical devices that are occasionally used for operations, especially for magnetic resonance images, preclude application of the system. ${ }^{26}$ By contrast, our RT3DE overcomes these limitations because of its lower cost, increased mobility, general patient access, and absence of radiation exposure.

Our RT3DE system provided adequate intraoperative images overall as an image-guided technology, but the spatial resolution of RT3DE still needs optimization to advance from simulation into a clinical setting. In addition, the transducer is too large to be able to apply it directly to the heart through a small incision because the operating field of instruments is restricted. Therefore further technologic development of an RT3DE system, such as by design of a high-frequency minitransducer or transesophageal 
transducer, will probably be necessary to make minimal incision RT3DE-guided beating-heart surgery possible.

In summary, model experiments were performed as a first step in the development of the methods and equipment necessary to perform image-guided beating-heart surgery for reconstructive repair of ASDs and MVP. The results showed that RT3DE provides adequate imaging and anatomic detail to serve as the sole guide for the performance of surgical tasks. These initial experiments demonstrate the feasibility of beating-heart direct or patch closure of ASD and MVP without CPB.

\section{References}

1. Harken DE, Ellis LB, Ware PF, et al. The surgical treatment of mitral stenosis: valvuloplasty. $N$ Engl J Med. 1948;239:801-9.

2. Bailey CP. The surgical treatment of mitral stenosis (mitral commissurotomy). Dis Chest. 1949;15:377-97.

3. Gibbon JH Jr. Application of a mechanical heart and lung apparatus to cardiac surgery. Minn Med. 1954;37:171-85.

4. Zeitlhofer J, Asenbaum S, Spiss C, et al. Central nervous system function after cardiopulmonary bypass. Eur Heart J. 1993;14:885-90.

5. Bellinger DC, Wypij D, Kuban KC, et al. Developmental and neurological status of children at 4 years of age after heart surgery with hypothermic circulatory arrest or low-flow cardiopulmonary bypass. Circulation. 1999;100:526-32.

6. Fix J, Isada L, Cosgrove D, et al. Do patients with less than 'echoperfect' results from mitral valve repair by intraoperative echocardiography have a different outcome? Circulation. 1993;88(suppl II): II39-48.

7. von Ramm OT, Smith SW. Real time volumetric ultrasound imaging system. J Digit Imaging. 1990;3:261-6.

8. Suematsu Y, Takamoto S. Semi-automatic suturing device for minimally invasive cardiac surgery. Kyobu Geka. 2003;56:203-6.

9. Suematsu Y, Takamoto S, Kaneko Y, et al. Beating atrial septal defect closure monitored by epicardial real-time three-dimensional echocardiography without cardiopulmonary bypass. Circulation. 2003;107: 785-90.

10. McGahan JP, Anderson MW. Pulsed Doppler sonography as an aid in ultrasound-guided aspiration biopsy. Gastrointest Radiol. 1987;12: 279-84.

11. Cannon JW, Stoll JA, Salgo IS, et al. Real time 3-dimensional ultrasound for guiding surgical tasks. Comput Aided Surg. 2003;8:82-90.

12. Maisano F, Torracca L, Oppizzi M, et al. The edge-to-edge technique: a simplified method to correct mitral insufficiency. Eur J Cardiothorac Surg. 1998;13:240-5.

13. Alfieri O, Maisano F, De Bonis M, et al. The double-orifice technique in mitral valve repair: a simple solution for complex problems. $J$ Thorac Cardiovasc Surg. 2001;122:674-81.

14. Pfister AJ, Zaki MS, Garcia JM, et al. Coronary artery bypass without cardiopulmonary bypass. Ann Thorac Surg. 1992;54:1085-92.

15. Suematsu Y, Ohtsuka T, Miyaji K, et al. Right heart mini-pump bypass for coronary artery bypass grafting: experimental study. Eur J Cardiothorac Surg. 2000;18:276-81.

16. Magni G, Hijazi ZM, Pandian NG, et al. Two- and three-dimensional transesophageal echocardiography in patient selection and assessment of atrial septal defect closure by the new DAS-Angel Wings device: initial clinical experience. Circulation. 1997;96:1722-8.

17. Chen C, Schneider B, Koschyk D, et al. Biplane transesophageal color Doppler echocardiography for assessment of mitral valve area with mitral inflow jet widths. J Am Soc Echocardiogr. 1995;8:121-31.

18. Austin EH. Editorial: transcatheter closure of atrial septal defects. J Thorac Cardiovasc Surg. 2000;120:1032-3.

19. Allen DS, Graham EA. Intracardiac surgery-a new method. JAMA. 1922;79:1028-30.

20. Wilson WG. Studies in experimental mitral obstruction in relation to the surgical treatment of mitral stenosis. Br J Surg. 1930;18:259-74.
21. Sogawa M, Moro H, Tsuchida M, Shinonaga M, Ohzeki H, Hayashi J. Development of an endocardioscope for repair of an atrial septal defect in the beating heart. ASAIO J. 1999;45:90-3.

22. Downing SW, Herzog WA Jr, McLaughlin JS, Gilbert TP. Beatingheart mitral valve surgery: preliminary model and methodology. J Thorac Cardiovasc Surg. 2002;123:1141-6.

23. Takuma S, Cardinale C, Homma S. Real-time three-dimensional stress echocardiography: a review of current applications. Echocardiography. 2000;17:791-4.

24. Lange A, Palka P, Burstow DJ, Godman MJ. Three-dimensional echocardiography: historical development and current applications. $J$ Am Soc Echocardiogr. 2001;14:403-12.

25. Sugeng L, Weinert L, Thiele K, Lang RM. Real-time three-dimensional echocardiography using a novel matrix array transducer. Echocardiography. 2003;20:623-35.

26. Woodard EJ, Leon SP, Moriarty TM, Quinones A, Zamani AA, Jolesz FA. Initial experience with intraoperative magnetic resonance imaging in spine surgery. Spine. 2001;26:410-7.

\section{Discussion}

Dr Patrick McCarthy (Chicago, Ill). Dr Suematsu and his colleagues deserve credit for performing these elegant studies to test the feasibility of using RT3DE to bring about intracardiac repair in a beating-heart model without CPB. This is a worthwhile goal. Everything we do in cardiothoracic surgery eventually reduces down to risk versus benefit and safety issues for the patient.

The benefit of the technique the authors describe is to reduce the risk of CPB. At least in the setting of ASD repair and mitral valve repair, this is not in itself a major source of morbidity. Therefore their echocardiography-guided procedure has to be exceptionally safe to show a benefit. On the basis of my own experience with RT3DE and the images that the authors have provided me, the spatial resolution of the 3D echocardiography is better than that of 2DE, as shown in their article. However, considering that damage to the atrioventricular node could occur during ASD repair and leaflet trauma or incorrect suturing, including embolization, which could occur during mitral valve surgery, then a surgeon would be reluctant to accept essentially fuzzy images to guide his or her operation, and that leads me to 2 questions.

First, are there any anticipated further improvements pending in the RT3DE that would make this more reproducible?

Dr Suematsu. If the surgical quality of the echocardiographyguided maneuver is similar to that of conventional surgical intervention, we believe that is much less invasive than conventional operations. The transducer we used in this study worked well in the water tank. However, the image quality (ie, the spatial resolution of this probe) is still suboptimal for detailed imaging of intracardiac structures and surgical devices. In addition, the size of the transducer is too large to apply it to the clinical setting. Therefore further technologic development of an RT3DE system, such as by design of a transesophageal transducer or a mini-3D transducer, will probably be necessary for in vivo use, and our colleagues have been developing that.

From a different point of view, we also need to develop surface coating of the device, in other words, an echocardiographyfriendly surgical device. In a preliminary study we examined sandblasting for echocardiographic coating of the surgical device, and that was pretty good, but we could not prevent side-lobe artifacts and shadowing completely. Therefore future development of surface coating for metallic devices or instrument designs 
compatible with echocardiographic imaging would be beneficial for a 3D echocardiography-guided image.

Dr McCarthy. The second question is as follows: do you anticipate clinical trials beginning with this technique soon or do you have further milestones before you begin that?

Dr Suematsu. We have now just started animal experiments with a pig model. As a first step, we focused on ASD creation, and the result was successful. As a next step, we are planning to perform ASD closure by using this modality. Unfortunately, we have no plan to use it clinically for the purpose of image-guided operations, but we also have just started evaluating RT3DE navigation in a clinical setting.
Dr D. Craig Miller (Stanford, Calif). Did you say that a 3D or 4D matrix transesophageal probe will be available soon? This would be impressive because the current Philips 7500 surface probe is huge.

Dr Suematsu. Yes. I am not sure when that system is available, but in the next 2 or 3 years, that system will be available.

Dr Craig R. Smith (New York, NY). I have just one comment. It seems to me that you are using the echocardiography in this setup to control the movement of clunky endoscopic instrument tips. If you could convert the echocardiographic information into one more source of sensory input to the brain of the da Vinci robot, you might really have something.

Access to The Journal of Thoracic and Cardiovascular Surgery Online is reserved for print subscribers!

Full-text access to The Journal of Thoracic and Cardiovascular Surgery Online is available for all print subscribers. To activate your individual online subscription, please visit The Journal of Thoracic and Cardiovascular Surgery Online, point your browser to http://www.mosby.com/jtcvs, follow the prompts to activate your online access, and follow the instructions. To activate your account, you will need your subscriber account number, which you can find on your mailing label (note: the number of digits in your subscriber account number varies from 6 to 10 ). See the example below in which the subscriber account number has been circled:

\section{Sample mailing label}

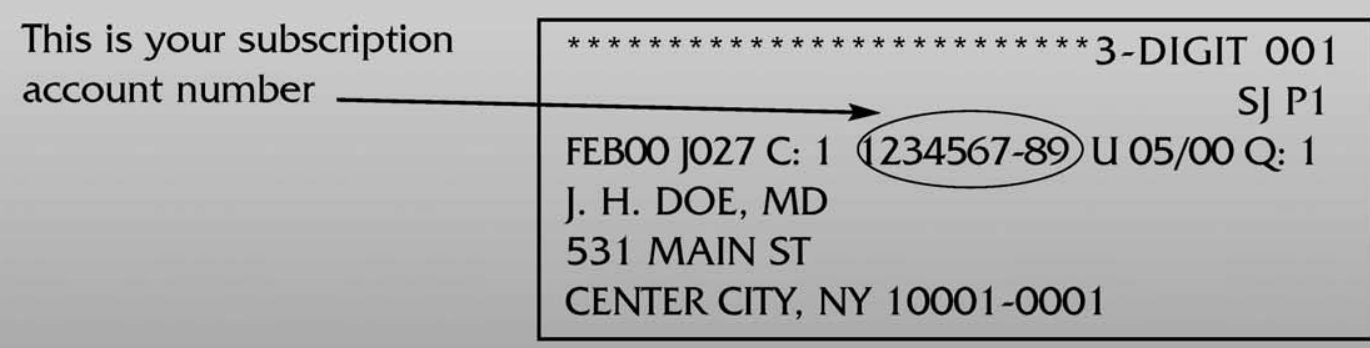

Personal subscriptions to The Journal of Thoracic and Cardiovascular Surgery Online are for individual use only and may not be transferred. Use of The Journal of Thoracic and Cardiovascular Surgery Online is subject to agreement to the terms and conditions as indicated online. 